\title{
Some properties of certain subclasses of multivalent integral operators
}

Deborah Olufunmilayo Makinde

Department of Mathematics, Obafemi Awolowo University, Ile-Ife 220005, Nigeria

E-mail: domakinde.comp@gmail.com, dmakinde@oauife.edu.ng

\begin{abstract}
For analytic function of the form $f_{i}(z)=z^{p}+\sum_{n=2} a_{n}^{i} z^{n}$, in the open unit disk, a class $\Gamma_{\alpha}^{p}\left(C_{1}, C_{2} ; \gamma\right)$ is introduced and some properties for $\Gamma_{\alpha}^{p}\left(C_{1}, C_{2} ; \gamma\right)$ of $f_{i}(z)$ in relation to coefficient bounds, convex conbination and convolution were obtained.
\end{abstract}

2010 Mathematics Subject Classification. $\mathbf{3 0 C 4 5}$

Keywords. Analytic, Multivalence, Coefficient bound, Convolution, Convex combination, Integral operator.

\section{Introduction}

Let $A$ denotes the class of functions of the form $f(z)=z+\sum_{n=2}^{\infty} a_{n} z^{n}, U=\{z \in C:|z|<1\}$ normalized with $f(0)=f^{\prime}(0)-1=0$ in the open disk, $U=\{z \in C:|z|<1\}$. In [8], Seenivasagan gave a condition for the univalence of the integral operator

$$
F_{\alpha, \beta}(z)=\left\{\beta \int_{0}^{z} t^{\beta-1} \prod_{t=1}^{k}\left(\frac{f_{i}(z)}{t}\right)^{\frac{1}{\alpha}} d t\right\}^{\frac{1}{\beta}}
$$

where $f_{i}(z)$ is defined by

$$
f_{i}(z)=z+\sum_{n=2}^{\infty} a_{n}^{i} z^{n}
$$

while Makinde and Opoola in [5] obtained a condition for the starlikeness of the function

$$
F_{\alpha}(z)=\int_{0}^{z} \prod_{t=1}^{k}\left(\frac{f_{i}(z)}{t}\right)^{\frac{1}{\alpha}} d t, \quad \alpha \in C
$$

where $f_{i}(z)$ is denoted by $(1.1)$.

Also, Xiao-Feili et al introduced the class $L_{1}^{*}\left(\beta_{1}, \zeta_{2}, \lambda\right)$ which is a subclass of $A$ such that

$$
L_{1}^{*}\left(\beta_{1}, \beta_{2}, \lambda\right)=\left\{f \in A:\left|\frac{f^{\prime}(z)-1}{\beta_{1} f^{\prime}(z)+\beta_{2}}\right| \leq \lambda\right\}, 0 \leq \beta_{1} \leq 1,0<\beta_{2} \leq 1 ; 0<\lambda \leq 1
$$

for some $\beta_{1} \beta_{2}$ and some real $\lambda$. He further denoted $T$ as the subclass of $A$ consisting of the function of the form:

$$
f(z)=z-\sum_{n=2}^{\infty} a_{n} z^{n}, \quad a_{n} \geq 0
$$

Tbilisi Mathematical Journal 7(2) (2014), pp. 79-83.

Tbilisi Centre for Mathematical Sciences.

Received by the editors: 18 June 2014

Accepted for publication: 17 November 2014 
and $L^{*}\left(\beta_{1}, \beta_{2}, \lambda\right)$ denotes the subclass of $L_{1}^{*}\left(\beta_{1}, \beta_{2}, \lambda\right)$ which is given by

$$
L^{*}\left(\beta_{1}, \beta_{2}, \lambda\right)=L_{1}^{*}\left(\beta_{1}, \beta_{2}, \lambda\right) \bigcap T
$$

for some real number $0 \leq \beta_{1} \leq 1,0<\beta_{2} \leq 1 ; 0<\lambda \leq 1$. The class $L^{*}\left(\beta_{1}, \beta_{2}, \lambda\right)$ was studied by Kim and Lee in [4], see also [1, 2, 9]. Moreover, Makinde and Oladipo in [7] introduced and studied the class $L_{\alpha}\left(\zeta_{1}, \zeta_{2}, \lambda\right)$.

Now, we defined $f_{i}(z)$ by

$$
f_{i}(z)=z^{p}+\sum_{n=2}^{\infty} a_{n}^{i} z^{n}
$$

Let $F_{\alpha}(z)$ be defined by $(1.2)$, then

$$
\frac{z F_{\alpha}^{\prime \prime}(z)}{F_{\alpha}^{\prime}(z)}=\sum_{i=1}^{k} \frac{1}{\alpha}\left(\frac{z f_{i}^{\prime}(z)}{f_{i}(z)}-1\right)
$$

Let $G(z)$ be denoted by

$$
G(z)=\sum_{i=1}^{k} \frac{1}{\alpha}\left(\frac{z f_{i}^{\prime}(z)}{f_{i}(z)}-1\right)
$$

We define

$$
\Gamma_{\alpha}^{p}\left(C_{1}, C_{2}, \gamma\right)=\left\{f_{i} \in A\left|\frac{G(z)+\frac{1}{\alpha}-1}{\zeta_{1}\left(G(z)+\frac{1}{\alpha}\right)+\zeta_{2}}\right| \leq \gamma\right\}
$$

for some complex $\zeta_{1}, \zeta_{2}, \alpha$ and for some real $\gamma, 0 \leq\left|\zeta_{1}\right| \leq 1,0<\left|\zeta_{2}\right| \leq 1,|\alpha| \leq 1$ and $0<\gamma \leq 1$.

\section{Main results}

Theorem 2.1. Let $f_{i}(z)$ be as in (1.3) and $F_{\alpha}(z)$ be as in (1.2). Then, $f_{i}(z)$ is in the class $\Gamma_{\alpha}^{p}\left(C_{1}, C_{2} ; \gamma\right)$ if and only if:

$$
\sum_{i=1}^{n} \sum_{k=p+1}^{\infty}\left\{k\left[\left(1+\gamma C_{1}\right)+\alpha\left(\gamma C_{2}-1\right)\right]\right\}\left|a_{k}^{i}\right| \leq \gamma\left|C_{1} p+\alpha C_{2}\right|-|p-\alpha|,
$$

$0 \leq C_{1} \leq 1,0<C_{1} \leq 1,0<\alpha \leq p$.

Proof. Using equations (1.1), (1.2) we have:

$$
\begin{aligned}
\left|\frac{G(z)+\frac{1}{\alpha}-1}{C_{1}\left(G(z)+\frac{1}{\alpha}\right)+C_{2}}\right| & =\left|\frac{\sum_{i=1}^{n}(p-\alpha)+\sum_{k=p+1}^{\infty}(k-\alpha) a_{k}^{i} z^{k-p}}{\sum_{i=1}^{n}\left(C_{1} p+\alpha C_{2}+\sum_{k=p+1}^{\infty}\left(k C_{1}+\alpha C_{2}\right) a_{k}^{i} z^{k-p}\right)}\right| \\
& \leq \frac{|p-\alpha|+\sum_{i=1}^{n} \sum_{k=p+1}^{\infty}(k-\alpha)\left|a_{k}^{i}\right|}{\left|C_{1} p+\alpha C_{2}\right|-\sum_{i=1}^{n} \sum_{k=p+1}^{\infty}\left(k C_{1}+\alpha C_{2}\right)\left|a_{k}^{i}\right|} .
\end{aligned}
$$

Let $f_{i}(z)$ satisfies the inequality $(2.1)$ then $f_{i}(z) \in \Gamma_{\alpha}^{p}\left(C_{1}, C_{2}\right)$.

Conversely, let $f_{i}(z) \in \Gamma_{\alpha}^{p}\left(C_{1}, C_{2}\right)$ then

$$
\sum_{i=1}^{n} \sum_{k=p+1}^{\infty}\left\{k\left[\left(1+\gamma C_{1}\right)+\alpha\left(\gamma C_{2}-1\right)\right]\right\}\left|a_{k}^{i}\right| \leq\left|C_{1} p+\alpha C_{2}\right|-|p-\alpha|
$$


Corollary 2.2. If $f_{i}(z) \in \Gamma_{\alpha}^{p}\left(C_{1}, C_{2} ; \gamma\right)$, we have:

$$
\sum_{i=1}^{n} \sum_{k=p+1}^{\infty}\left|a_{k}^{i}\right| \leq \frac{\left|C_{1} p+\alpha C_{2}\right|-|p-\alpha|}{k\left[\left(1+\gamma C_{1}\right)+\alpha\left(\gamma C_{2}-1\right)\right]}
$$

Theorem 2.3. Let $f_{i}(z) \in \Gamma_{\alpha}^{p}\left(C_{1}, C_{2}\right)$ and $g_{i}(z)$ defined by $g_{i}(z)=z^{p}+\sum_{k=p+1}^{\infty} b_{k}^{i} z^{k}$ be in the same $\Gamma_{\alpha}^{p}\left(C_{1}, C_{2}\right)$. Then the function $h_{i}(z)$ defined by

$$
h_{i}(z)=\left(1-\lambda f_{i}(z)+\lambda g_{i}(z)=z^{p}+\sum_{k=p+1}^{\infty} C_{k}^{i} z^{k}\right.
$$

is also in the class $\Gamma_{\alpha}^{p}\left(C_{1}, C_{2}\right)$ where

$$
C_{k}^{i}=(1-\lambda) a_{k}^{i}+\lambda b_{k}^{i}, \quad 0 \leq \lambda \leq 1 .
$$

Proof. Suppose each of $f_{i}(z)$ and $g_{i}(z)$ in the $\Gamma_{\alpha}^{p}\left(C_{1}, C_{2} ; \lambda\right)$. Then we have

$$
\begin{aligned}
\sum_{i=1}^{n} \sum_{k=p+1}^{\infty}\left\{k\left[\left(1+\gamma C_{1}\right)+\alpha\left(\gamma C_{2}-1\right)\right]\right\}\left|C_{k}^{i}\right| & =\sum_{i=1}^{n} \sum_{k=p+1}^{\infty}\left\{k\left[\left(1+\gamma C_{1}\right)+\alpha\left(\gamma C_{2}-1\right)\right]\right\}\left|(1-\lambda) a_{k}^{i}+\lambda b_{k}^{i}\right| \\
& =(1-\lambda) \sum_{i=1}^{n} \sum_{k=p+1}^{\infty}\left\{k\left[\left(1+\gamma C_{1}\right)+\alpha\left(\gamma C_{2}-1\right)\right]\right\}\left|a_{k}^{i}\right| \\
& +\lambda \sum_{i=1}^{n} \sum_{k=p+1}^{\infty}\left\{k\left[\left(1+\gamma C_{1}\right)+\alpha\left(\gamma C_{2}-1\right)\right]\right\}\left|b_{k}^{i}\right| \\
& \leq(1-\lambda)\left(\left|C_{1} p+\alpha C_{2}\right|-|p-\alpha|\right) \\
& +\lambda\left(\left|C_{1} p+\alpha C_{2}\right|-|p-\alpha|\right) \\
& =\left|C_{1} p+\alpha C_{2}\right|-|p-\alpha|
\end{aligned}
$$

which shows that the convex combination of $f_{i}(z)$ and $g_{i}(z)$ is in the class $\Gamma_{\alpha}^{p}\left(C_{1}, C_{2} ; \gamma\right)$. $\quad$ Q.E.D.

Theorem 2.4. Let $f_{i}(z)$ be as in $(3)$ and $F_{\alpha}(z)$ be as in $(1.2)$ then the function $C_{i}(z)$ defined by

$$
C_{i}(z)=z^{p}+\sum_{k=p+1}^{\infty} a_{k}^{i} b_{k}^{i} z^{k}
$$

is in the class $\Gamma_{\alpha}^{p}\left(C_{1}, C_{2} ; \gamma\right)$ if and only if

$$
\sum_{i=1}^{n} \sum_{k=p+1}^{\infty}\left\{k\left[\left(1+\gamma C_{1}\right)+\alpha\left(\gamma C_{2}-1\right)\right]\right\}\left|a_{k}^{i} b_{k}^{i}\right| \leq \gamma\left|C_{1} p+\alpha C_{2}\right|-|p-\alpha|,
$$

$0 \leq C_{1} \leq 1,0<C_{1} \leq 1,0<\alpha \leq p$.

Proof. Following the procedure of the proof of the Theorem 1, we obtain the result. 
Theorem 2.5. Let the function $\psi_{i}(z)$ be in the class $\Gamma_{\alpha}^{p}\left(C_{1}, C_{2} ; \gamma\right)$ and the function $\zeta_{i}(z)$ defined by

$$
\zeta_{i}(z)=z^{p}+\sum_{k=p+1}^{\infty} A_{k}^{i} B_{k}^{i} z^{k}
$$

be in the same $\Gamma_{\alpha}^{p}\left(C_{1}, C_{2} ; \gamma\right)$. Then the function $H(z)$ defined by

$$
H(z)=(1-\lambda) \zeta_{i}(z)+\lambda \psi_{i}(z)=z^{p}+\sum_{k=p+1}^{\infty} C_{k}^{i}
$$

is also in the class $\Gamma_{\alpha}^{p}\left(C_{1}, C_{2} ; \gamma\right)$, where

$$
C_{k}^{i}=(1-\lambda) a_{k}^{i} b_{k}^{i}+\lambda A_{k}^{i} B_{k}^{i}, \quad 0 \leq \lambda \leq 1 .
$$

Proof. The proof of the theorem is similar to that of Theorem 2, thus, we omit the proof. $\quad$ Q.E.D.

Corollary 2.6. Let $f_{i}(z)$ be as in (1.3) and $F_{\alpha}$ be as in (1.4). Then $f_{i}(z)$ is in the class $\Gamma_{\alpha}^{p}\left(C_{1}, C_{2} ; \gamma\right)$ if and only if

$$
\sum_{i=1}^{n} \sum_{k=p+1}^{\infty}\left\{k\left[\left(1+\gamma C_{1}\right)+\alpha\left(\gamma C_{2}-1\right)\right]\right\}\left|a_{k}^{i}\right| \leq \gamma\left|C_{1} p+\alpha C_{2}\right|-|p-\alpha|
$$

$0 \leq C_{1} \leq 1,0<C_{1} \leq 1,0<\alpha \leq p$

\section{References}

[1] O. Altintas, and S. Owa, On soem subclassess of univalent functions with negative coefficient, Pusan Kyongnam Math. J. 4 (1988) 41-46.

[2] D. Hur and G.H. Oh, On certain class of analytic functions with negative ciefficients, Pusan Kyongnam Math. J. 5(19890), 69-80.

[3] S. Kanas and F.Ronnig, Unifromly Starlike and conves functions and other related classes of univalent functions, Ann Universitym Narue Curie-Sklodowska Section A,53(1999), 95-105.

[4] H.S. Kim and S.K. Lee, On soem classes of univalent functions, Math Japan, 32(987), 781-796.

[5] D.O Makinde and T.O. Opoola, On sufficient condition fo starlikeness, General Mathematics 18(3) 2010, 30-35.

[6] D.O. Makinde, On a certain family of meromorphic functions with positive coefficients, Acta Universitatis Apulensis 36 2013, 203-208.

[7] D.O. Makinde and A.T. Oladipo, Some properties of certain subclasses of univalent integral operator, Scientia Magna 9(1) 2013, 80-88 
[8] N. Seenivasagan, Sufficient conditions for univalence, Applied Mathemaitics E-notes, 8,2008, 30-35.

[9] F. Silverman, Univalent functions with negative coefficients, Proc. Amer. Math. Soc, 51 (1975),109-116.

[10] X.-F. Li and A.-P. Wang, Some properties of some subclassess of univalent functions, Applied Mathematical Sciences, 6(54) (2012) 2687-2693. 